\title{
Enhancement of Tropical Land Cover Mapping with Wavelet-Based Fusion and Unsupervised Clustering of SAR and Landsat Image Data
}

\author{
Jacqueline Le Moigne', Nadine Laporte ${ }^{2}$, Nathan S. Netanyahu ${ }^{3}$
}

1. Applied Information Sciences Branch, NASA Goddard Space Flight Center, Greenbelt, MD 20771; lemoigne@backserv.gsfc.nasa.gov

2. University of Maryland, Geography Dept, College Park, MD 20742 USA; nlaporte@geog.umd.edu

3. Bar-Ilan University, Ramat-Gan 52900, Israel; nathan@cs.biu.ac.il, and UMIACS/University of Maryland, College Park, MD 20742 USA; nathan@cfar.umd.edu

\begin{abstract}
The characterization and the mapping of land cover/land use of forest areas, such as the Central African rainforest, is a very complex task. This complexity is mainly due to the extent of such areas and, as a consequence, to the lack of full and continuous cloud-free coverage of those large regions by one single remote sensing instrument. In order to provide improved vegetation maps of Central Africa and to develop forest monitoring techniques for applications at the local and regional scales, we propose to utilize multi-sensor remote sensing observations coupled with in-situ data. Fusion and clustering of multi-sensor data are the first steps towards the development of such a forest monitoring system. In this paper, we will describe some preliminary experiments involving the fusion of SAR and Landsat image data of the Lopé Reserve in Gabon. Similarly to previous fusion studies, our fusion method is wavelet-based. The fusion provides a new image data set which contains more detailed texture features and preserves the large homogeneous regions that are observed by the Thematic Mapper sensor. The fusion step is followed by unsupervised clustering and provides a vegetation map of the area.
\end{abstract}

Keywords: image fusion, land cover mapping, wavelet representation, unsupervised clustering, remote sensing imagery.

\section{INTRODUCTION}

Due to the extent of forest areas such as the Central African rainforest, and as a consequence of the lack of full and continuous cloud-free coverage of those large regions by one single remote sensing instrument, multi-sensor remote sensing observations coupled with in situ data are the only way to survey the 1.8 Million $\mathrm{km}^{2}$ rainforest of the Congo Basin in a consistent fashion. The most recent maps of the region are from the 1990's and were derived from $1 \mathrm{~km}$ AVHRR-LAC data $^{1,2}$. These products do not reflect the last decade of land use change in the region, which we know to have been substantive. Moreover, due to limited optical remote sensing data sets, the NASA Landsat Pathfinder project was only able to produce land cover maps for two countries, the Democratic Republic of Congo (DRC) and the Central African Republic (CAR). Current mappings based on JERS-1 data by JPL ${ }^{2,3}$ are still formative and do not accurately depict the extent of forest lands converted to agriculture. It is therefore critical to develop hybrid approaches combining RADAR and optical remote sensing to monitor human pressure on forested lands and to estimate amounts of above-ground forest and agricultural biomass.

The fusion and clustering of multi-sensor data are the first steps towards the development of such a forest monitoring system. For most areas, cloud-free high-resolution data such as Landsat data is not available. Therefore, the main goal of our research is to fuse RADAR and low-resolution optical data such as AVHRR or MODIS data, and investigate if such a fusion enables to create classification maps of a quality at least equivalent to those created by high-resolution data. Such a process would enable to fill the gaps of high-resolution land cover maps at locations where high-resolution data such as Landsat are not available. Figure 1 illustrates this idea: our future work will involve the fusion of JERS data with MODIS data and will compare the clustering or the classification of these fused data to those of Landsat data alone. In this paper, we describe more preliminary experiments by comparing the mapping of data obtained by fusion of SAR and Landsat image data of the Lopé Reserve in Gabon compared to the mapping obtained from the same Landsat data alone. These tests represent a "school example" of the future fusion needed for our forest monitoring system.

Similarly to previous fusion studies ${ }^{4-6}$, our fusion method is wavelet-based, integrating high-frequency components of the higher spatial resolution SAR data and low-frequency components of the lower spatial resolution Landsat-TM data. The fusion provides a new image data set which contains more detailed texture features, that should help improve the final 
mapping. At the same time, the fusion preserves the large homogeneous regions that are observed by the Thematic Mapper sensor. Wavelet-based fusion is described in section 2.

The fusion step is followed by unsupervised clustering, e.g. K-means or ISODATA, that provides a vegetation map of the area. The unsupervised clustering is described in section 3. Original Landsat data and fused data are processed according to this clustering scheme and the resulting mappings are evaluated qualitatively. Results are presented in section 4 .

\section{IMAGE FUSION FOR CLASSIFICATION}

Fusion of multi-sensor data may be performed either before the classification step with a pixel-level image fusion technique, or as a post-processing by combining multiple classification results at the feature- or decision-level. In this work, we propose to perform the fusion before the classification. A general survey of pixel-level image fusion techniques can be found in Pohl et $\mathrm{al}^{4}$. Several statistical/ numerical methods have been proposed and include: arithmetic combinations, Principal Component Analysis (PCA), High Pass Filtering, Regression Variable Substitution (RVS), Canonical Variate Substitution, Component Substitution, and Wavelet Transforms. For our application, we will consider PCA, RVS, and wavelet transforms as well as hybrid techniques composed of these three basic methods. A brief description of those three techniques is given below, and the results described in this paper will be based on the use of a wavelet transform:

$>$ Principal Component Analysis (or PCA) is a method that enables a decrease in the number of channels (or bands) by reducing the inter-channel dependencies. Its principle is based on the computation of the covariance matrix and its diagonalization by finding corresponding eigenvalues and eigenvectors. The first eigenvector (or Principal Component) is a linear combination of the initial bands and usually contains more than $90 \%$ of the information contained in all channels. Two sets of data can then fused by concatenating all channels and performing a PCA transformation on all channels to keep only non-redundant information. This step is especially useful for classification purposes (e.g., neural network approaches). Various algorithms and parallel implementations of PCA are being studied independently 7.8

$>$ Regression Variable Substitution (or RVS), uses a multiple regression procedure to determine a linear combination (or replacement vector) of an image channel that can be replaced by another channel. Such a method was used successfully to fuse data from SeaWIFS and AVHRR'.

Wavelet Transforms describe images in the frequency domain at multiple spatial resolutions using a Multi-Resolution Analysis (MRA). Therefore, performing image fusion within a wavelet framework enables to fuse data selectively at various frequency components in the lower spatial resolutions, while preserving spectral information of higher spatial resolutions. Wavelet transforms have been used to fuse multispectral and panchromatic data ${ }^{5,6}$. In our investigation, we propose to utilize such a scheme where multi-sensor data will be fused during the reconstruction phase of the wavelet transform.

$>$ Hybrid Approaches combining PCA and wavelets or RVS and wavelets, that will be able to handle multiple spatial and radiometric resolution data, can also be utilized.

In the remainder of this paper, we will focus on wavelet-based image fusion.

\subsection{Wavelet Representation}

Briefly, a wavelet decomposition of any given signal (1-D or 2-D) is the process which provides a complete representation of the signal according to a well-chosen division of the time-frequency (1-D) or space-frequency (2-D) plane. Through iterative filtering by low-and high-pass filters, it provides information about low- and high-frequencies of the signal at successive spatial scales. See Daubechies ${ }^{10}$ and Mallat ${ }^{11}$ for more detail on wavelet transforms. Multi-resolution wavelets, that are largely used for data compression and browsing, are utilized here:

- first, as a way to bring multiple types of data to the same spatial resolution without losing significant information and without blurring the higher resolution data. Multi-resolution wavelet decomposition preserves most of all important features of the original data even at a lower resolution, especially global scale features such as rivers, roads, and lakes.

- second, for fusion purposes, further multi-resolution wavelet decomposition separates high and low-frequency components which are then recomposed differently in the reconstruction phase.

In our experiments, we will be using a Daubechies filter ${ }^{10}$ size 4 and a Mallat MRA " decomposition and reconstruction scheme. Figure 2 shows the principle of the MRA wavelet decomposition, with $\mathrm{L}$ representing a low-pass filter and $\mathrm{H}$ a high-pass filter.

\subsection{Wavelet-Based Fusion}

Figure 3 illustrates the wavelet-based fusion idea, where high- and low-resolution data are independently decomposed using the MRA scheme described in Figure 2. Then, components from both decompositions are combined during the reconstruction phase to create the new fused data. 
In this scheme, low-frequency information of the lowest spatial resolution data (i.e., Landsat data) are combined with high-frequency information of the highest resolution data (i.e., SAR data) in order to take simultaneous advantage of the higher spatial and spectral resolutions. In our experiments, the same Daubechies filter size 4 was used for both decomposition and reconstruction phases and for both types of data. But one can imagine using different filters for decomposition and reconstruction as well as for high- and low-resolution data, that would better preserve the spatial, spectral and textural properties of the data.

\section{UNSUPERVISED CLASSIFICATION}

The fusion stage is followed by unsupervised classification, in order to obtain a vegetation map of the area of interest. Such an approach is common when reliable training data (for supervised classification) are either scarce or expensive, and when relatively little a priori information about the data is available. Unsupervised clustering methods play a significant role in the pursuit of unsupervised classification (see, e.g., Richards and $\mathrm{Jia}^{12}$, for general background). Once a reliable clustering is arrived at, the user can then label the corresponding image segments to obtain a classified image.

Indeed, various unsupervised clustering schemes have been proposed and studied over the years ${ }^{13,14}$. In particular, classical methods such as K-means and ISODATA ${ }^{15}$, which are based on iterative computations of cluster means, have become standard also in the remote sensing community. (ISODATA is more involved than K-means, in the sense that it provides additional heuristic procedures (e.g., cluster merging and splitting) and some interactive features.) For the case study reported in this paper, we used ISOCLUS ${ }^{16}$, which is very similar to ISODATA. The number of clusters was set to 10 , and termination was applied either after 20 iterations or when a relative change in all of the cluster means did not exceed $1 \%$. The results obtained are presented and described in the following section.

We note that to further enhance our unsupervised clustering scheme, we have been developing a fast K-means module. The module is based on a recent improvement to Llyod's K-means algorithm ${ }^{17}$. The basic idea is to gain significant speedup by pre-processing the data points, such that, nearest neighbor computations become much more efficient. The module that is currently under way is aimed at gaining yet further speedup by employing the above K-means variant to merely a sample of the image points. Depending on certain distortion criteria, however, the sample may need to be refined (e.g., by attaching stronger weights to certain points), so as to obtain a more "representative" sample. We hope to employ successfully this module to the ongoing application reported in this paper. Furthermore, since many unsupervised clustering schemes bear strong similarity to the K-means algorithm -- this is reflected in the in basic, recurring computation of point distances to cluster means -- we will attempt to incorporate it in more comprehensive schemes, such as ISODATA.

\section{RESULTS}

\subsection{Description of the Test Datasets}

The data used for this study cover the area of the Lopé Reserve in Gabon, corresponding to a region of Central Africa included between longitudes 11 degrees East to 12 degrees East and a latitude between 0 degrees and -1 degree South. For this region, the low-resolution data is represented by a sub-image extracted from Landsat scene \#185-060, acquired on May Ist, 1988, with an original spatial resolution of 30 meters per pixel. The high-resolution data is extracted from a SAR image acquired from the "Mission Aéroportée Radar SAR" flown in 1994. The resolution of the SAR data is of 6 meters per pixel. For these first experiments, since we use the Landsat data set both as reference and as part of the fusion, we resampled it to 6 meters using the PCI software. But generally, this resampling will not be necessary since different levels of wavelet decomposition are used for both types of data. After resampling, the size of the images to be fused is $2048 \times 2048$. Figures 4 and 5 show the original SAR and Landsat scenes with the windows of interest extracted from each of them: these subimages are shown in Figures 6 and 7.

\subsection{Description of Land Cover Types}

Generally, for the Lopé area, nine different classes can be defined:

(1) Mountain Forest

(2) Old Forest or Mixed Forest or Marantaceaes Forest, pluri-stratum forest with at least 3 different tree heights

(3) Okoume Forest, mono-stratum forest with trees of similar diameters and heights (40 to $60 \mathrm{~m}$ high for adult trees)

(4) Riparian Forest or Galery Forest

(5) Savanna or Grasses, of height in the range of 1 to 1.5 meters

(6) Fern Savanna of height less than 1 meter

(7) Swampy Savanna with Shrubs

(8) Burnt Savanna 
(9) Bare Soil

(10) Pist or Road

For the area represented in Figures 6 and 7, only six types of land cover are represented:

(LI) Mountain Forest

(L2) Mixed Forest

(L3) Okoume Forest

(L4) Savanna or Grasses, of height in the range of 1 to 1.5 meters

(L5) Fern Savanna

(L6) Burnt Savanna

\subsection{Results}

As a first step, both SAR and Landsat data are decomposed using 4 levels of wavelet decomposition and a Daubechies filter size 4. Then, utilizing the same filter, the two data sets were fused with the scheme outlined in Figure 3 . The fused image is shown in Figure 9. After fusion, the 3 bands of both Landsat data and fused data were processed with the $\mathrm{PCl} / \mathrm{ISOCLUS}$ clustering. The initial number of clusters was set to 10 and after 20 iterations, the program returns 9 clusters for both the Landsat and the fused data set. Then, thematically, these nine clusters can be regrouped into the 6 types described in the previous section. The clustering step takes between 10 to 15 seconds on a Dell optiplex gx300 with two Intel PentiumIII CPUs et 512 MB RAM. Results are shown in Figures 10 and 11, where dark green,light green, orange, light blue, pink, and dark blue represent respectively Mountain Forest (type L1), Mixed Forest (L2), Okoume Forest (L3), Savanna (L4), Fern Savanna (L5), and Burnt Savanna (L6). Qualitatively, we can see that the results are similar, but that the fused clustering shows more localized details with differentiation of the different types of savannas in the right side of the image and a different clustering of Mountain Forest versus Mixed Forest in the left side of the image. These results will be evaluated quantitatively, once in-situ data become available.

\section{CONCLUSIONS AND FUTURE WORK}

The study presented in this paper is a case study towards a wavelet-based fusion and mapping of SAR and optical data that will enable to develop a forest monitoring system for applications at the local and regional scales. Future work will involve JERS and MODIS data compared to Landsat and to MODIS data alone, the investigation of different types of wavelet filters, improvements of the clustering scheme as well as a quantitative evaluation of the results using in-situ data.

\section{ACKNOWLEDGMENTS}

The authors wish to acknowledge the support of NASA under NRA-99-OES-06 on "Land Cover and Land Use Change Research" and under NRA-NAS2-37143 on "Research in Intelligent Systems." The authors would also like to thank Didier Devers for his help in preparing this paper.

\section{REFERENCES}

1. N. Laporte, S.J. Goetz, C.O. Justice, and M. Heinicke, "A New Land Cover Map of Central Africa Derived from Multi-Resolution, Multi-Temporal AVHRR Data," International Journal of Remote Sensing, 19(18), pps.3537-3550, 1998.

2. P. Mayaux, G. DeGrandi, J.P. Malingreau, "Central African Forest Cover Revisited: a Multi-Satellite Analysís," Remote Sensing of the Environment, 71, pps. 183-196, 2000.

3. G. DeGrandi, J.P. Malingreau, M. Leysen, "The ERS-1 Central Afrimosaic: A New Perspective in Radar Remote Sensing for Global Monitoring of Vegetation," IEEE Transactions on Geoscience and Remote Sensing, vol. 37, no. 3, pps 1730-1746, May 1999.

4. C. Pohl, and J.L. Van Genderen, "Multisensor Image Fusion in Remote Sensing: Concepts, Methods and Applications," International Journal of Remote Sensing, vol. 19, No. 5, pps. 823-854, 1998.

5. T. Ranchin, and L. Wald, "Data Fusion of Remotely Sensed Images Using the Wavelet Transform: the ARSIS Solution," Proceedings SPIE Wovelet Applications in Signal and Image Processing IV, 1997.

6. IGARSS 2000 Data Fusion Contest, http:// www.aris.sai.jrc.it/dfc/competition.html.

7. S. Kaewpijit, J. Le Moigne, and T. El-Ghazawi, "Finding the Dimensionality of Hyperspectral Data," SPIE Aerosense 200l, Algorithms for Multispectral, Hyperspectral, and Ultraspectral Imagery VII, Orlando, FL, April 16$20,2001$. 
8. T. El-Ghazawi, S. Kaewpijit, and J. Le Moigne, "Parallel Adaptive Reduction of Hyperspectral Data to its Intrinsic Dimensionality," Third IEEE International Conference on Cluster Computing, Cluster Computing '200I, Newport Beach, California, October 8-11, 2001.

9 J. Pinzon, D. Slayback, C. Tucker, "Wide Field-of-View Spectrometer Optics," Monthly Report: February, March. April, NASA/GSFC, Code 923, 1999.

10. I. Daubechies, Ten Lessons on Wovelets, CMBS-NSF Series Applications in Mathematics, SIAM, 1991.

11. S. Mallat, "A Theory for Multiresolution Signal Decomposition," IEEE Pattern Analysis and Machine Intelligence, vol. PAMI-11, no. 7, 1989.

12. J.A. Richards and X. Jia, Remote Sensing Digital Image Analysis, Springer, Berlin, 1999.

13. H. Yuan, S. Khorram, and X. Long Dai, "Applications of Simulated Annealing Minimization Technique to Unsupervised Classification of Remotely Sensed Data, "Proceedings of the IEEE International Geoscience and Remote Sensing Symposium, Hamburg, Germany, pp. 134--136, 1999.

14. J. Cihlar, R. Latifovic, and J. Beaubien "A Comparison of Clustering Strategies for Unsupervised Classification," Canadian Journal of Remote Sensing, Vol. 26, pp. 446--454, 2000.

15. J.T. Tou and R.C. Gonzales, Pattern Recognition Principles, Addison Wesley, 1974.

16. PCI (1999), EASY/PACE Software Package, Version 6.3. PCI Inc., Richmond Hill, Ontario, Canada.

17. T. Kanungo, D.M. Mount, N.S. Netanyahu, C. Piatko, R. Silverman, and A.Y. Wu, "The Analysis of a Simple Kmeans Cluetering Algorithm," Proceedings of the 16th Annual ACM Symposium on Computational Geometry, Hong Kong, China, pp. 100--109, 2000. 

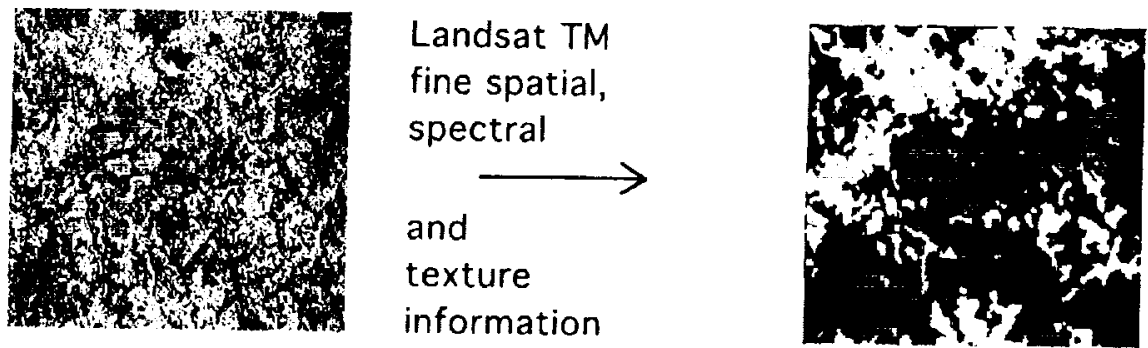

Forest

Non-forest

$\square$ Regrowth

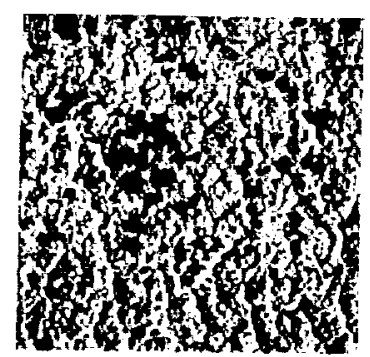

fine spatial and texture information from JERS- 1 image
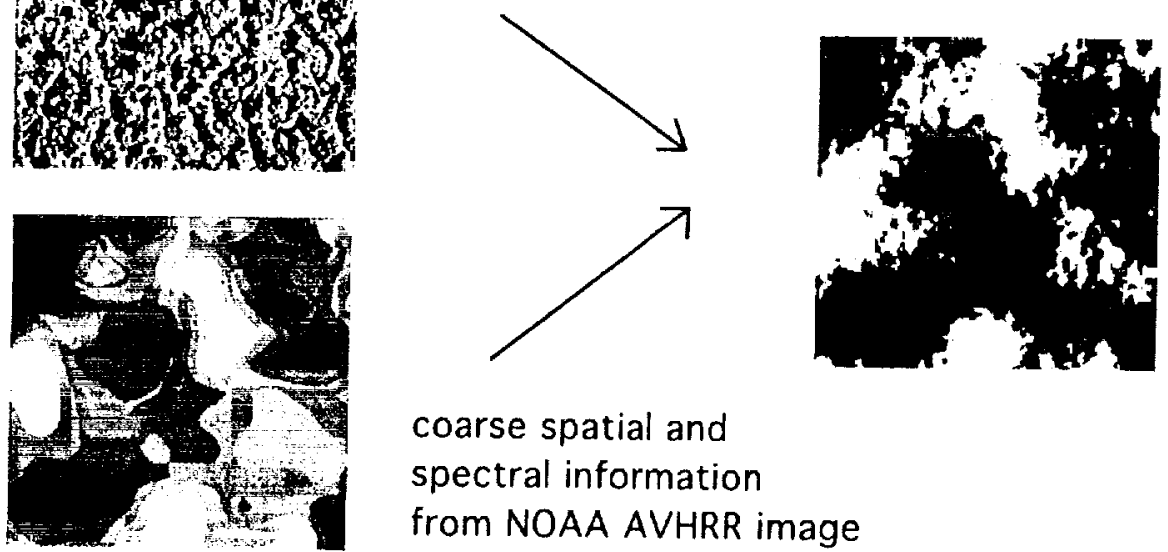

Forest

Non-forest

coarse spatial and spectral information from NOAA AVHRR image

Figure 1

Fusion Illustration

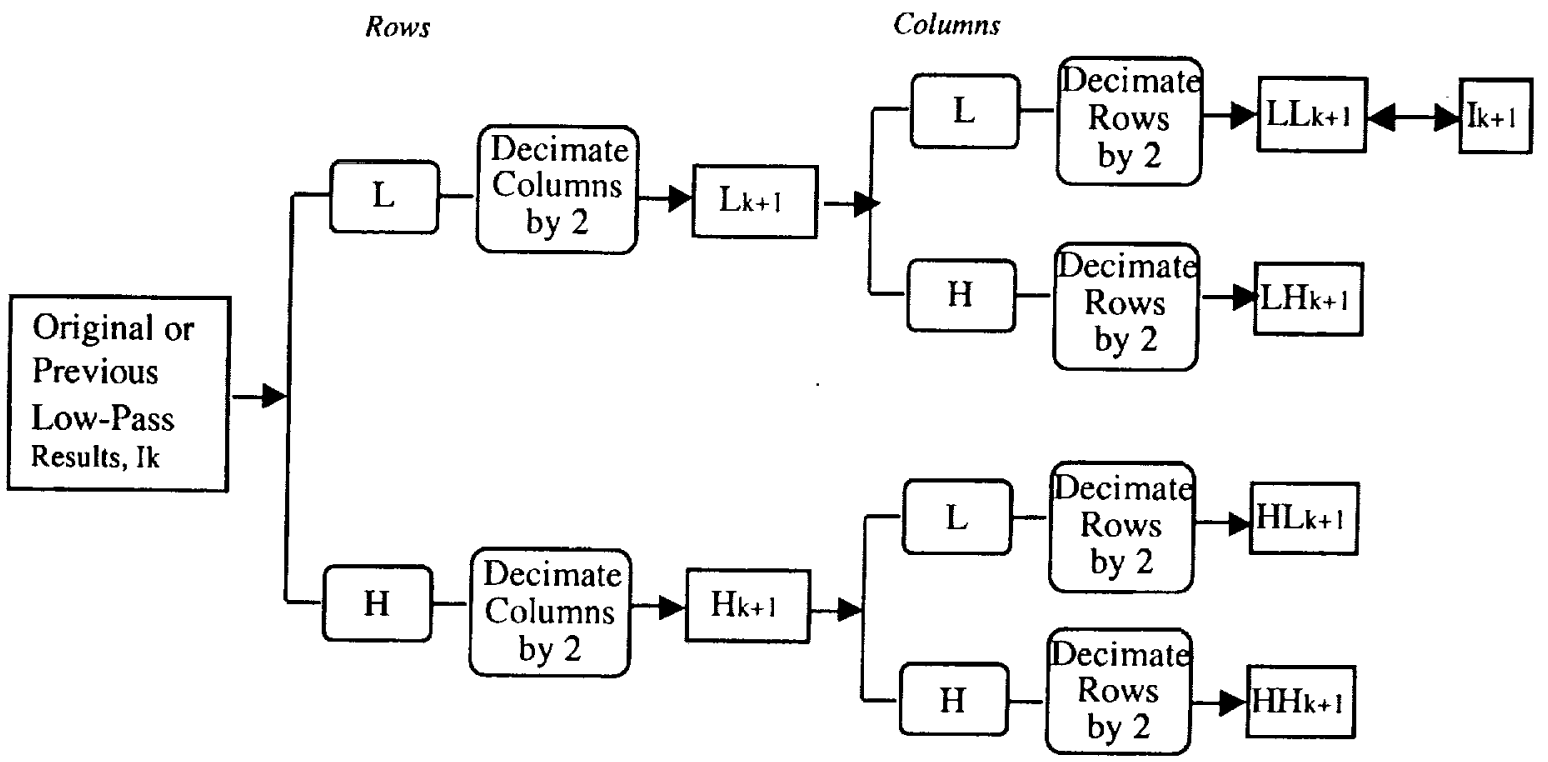

F represents the convolution of the input image by the filter $F$

Figure 2

Wavelet Decomposition 


\section{Wavelet-Based Fusion}

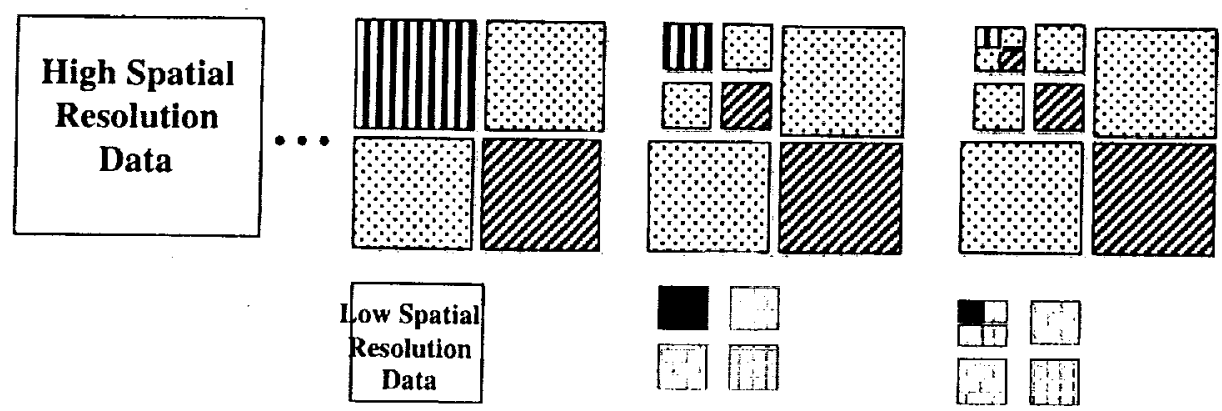

Decomposition

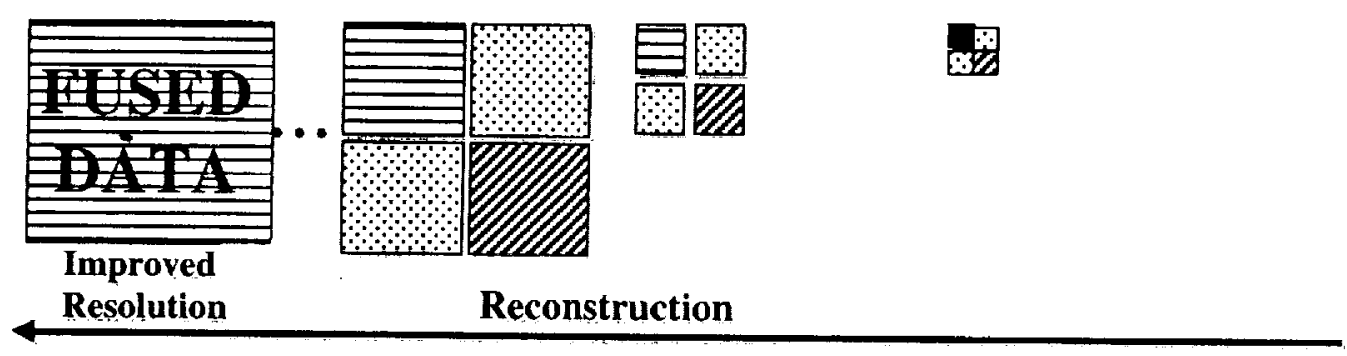

Figure 3

Wavelet-Based Image Fusion

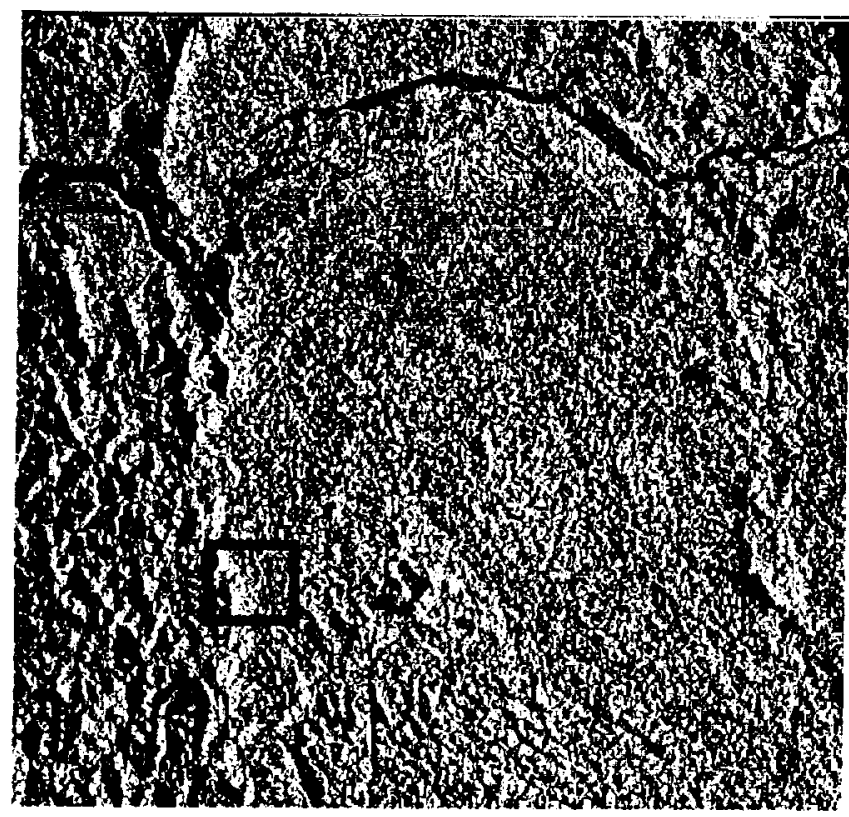

Figure 4

Original SAR Data with Window of Interest

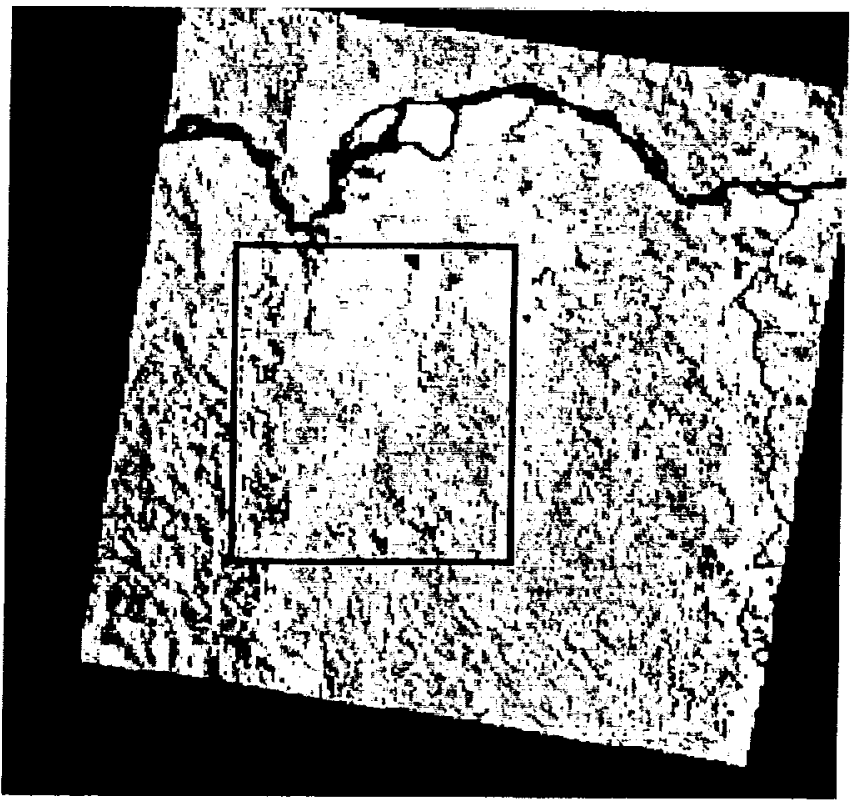

Figure 5

Original Landsat Data with Window of Interest 


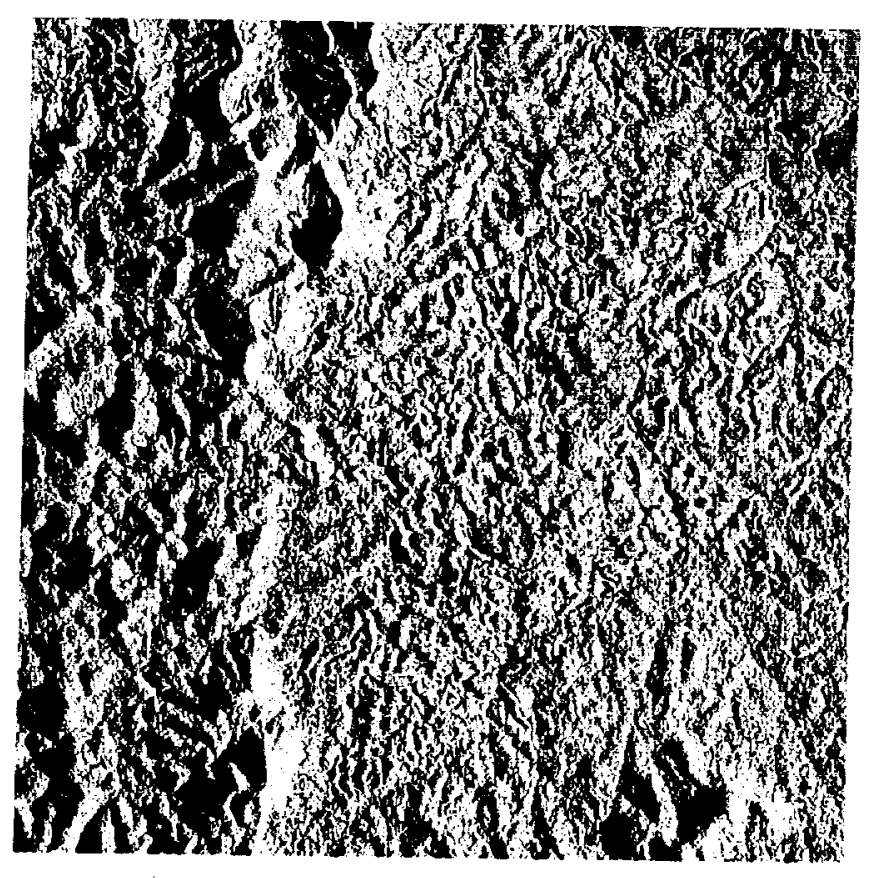

Figure 6

Window of Interest: 6 meters - SAR Data

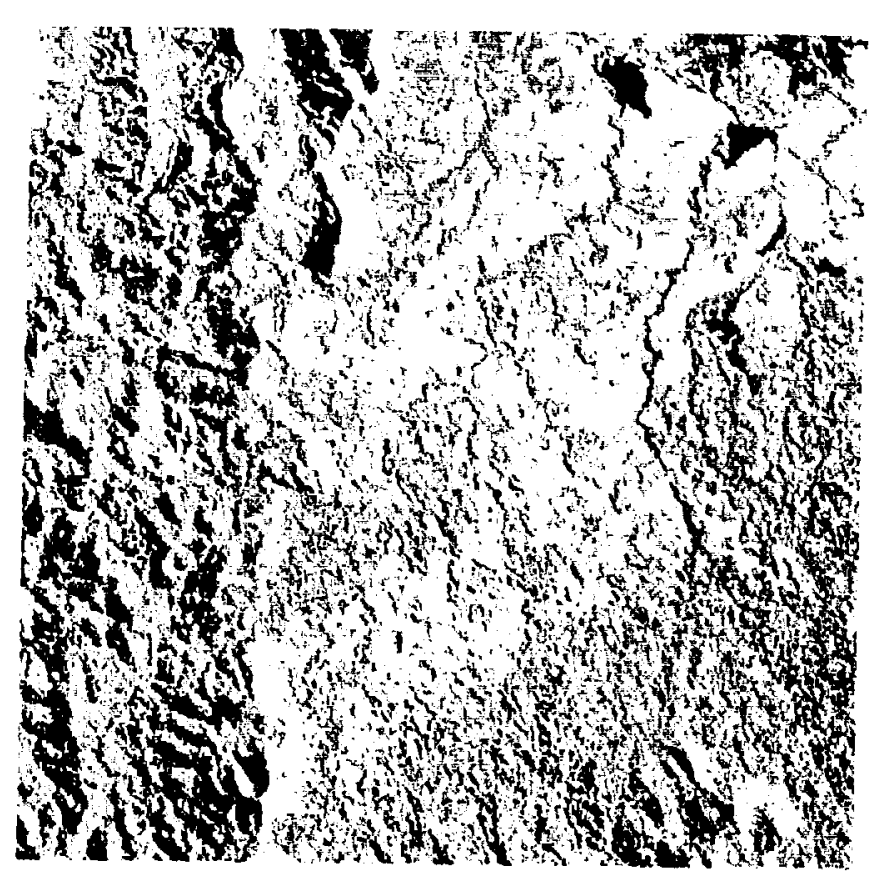

Figure 7

Window of Interest: Landsat Data Resampled to 6 meters

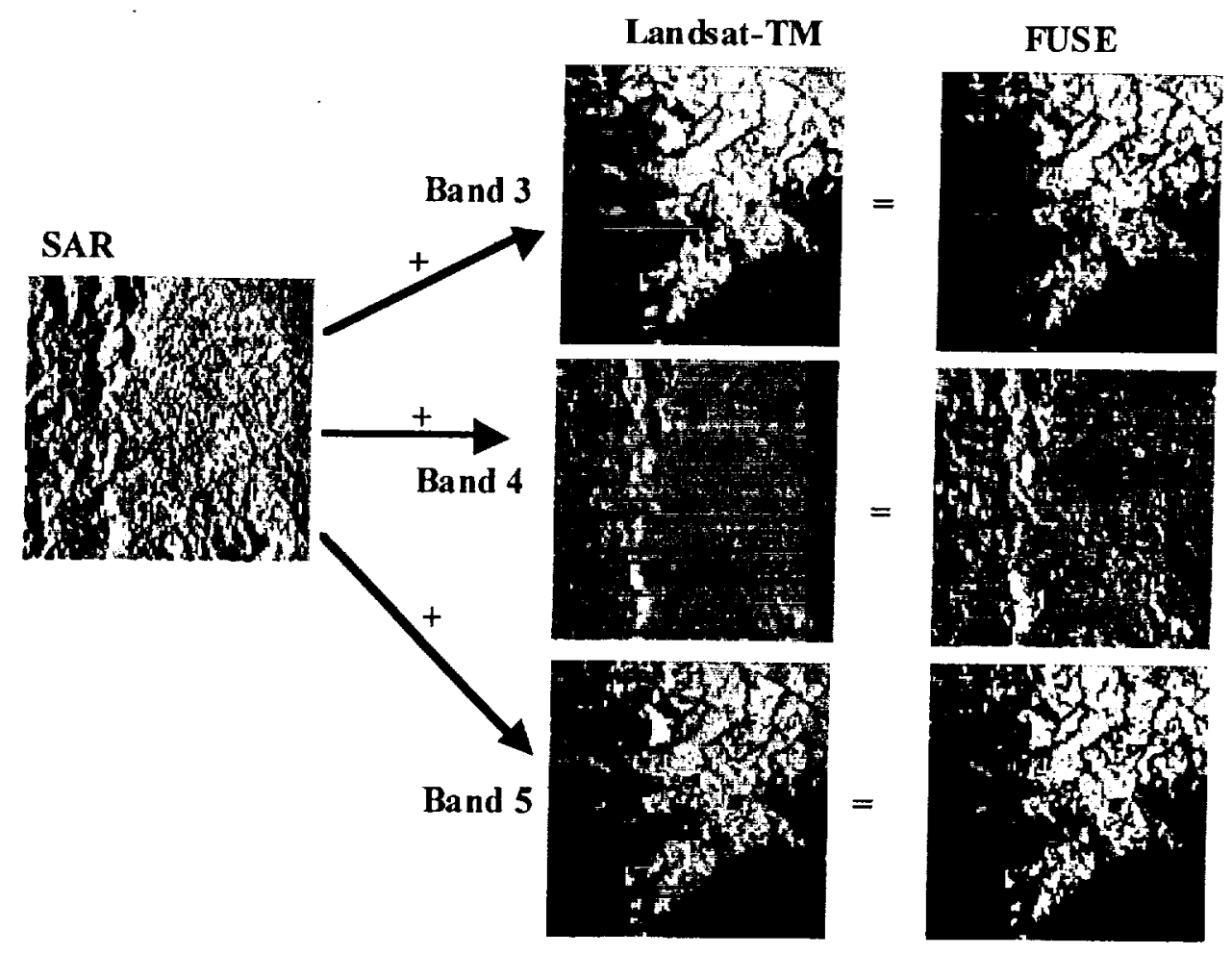

Figure 8

Wavelet-Based Fusion of 6m-SAR Data with Bands3,4,5 of Resampled 6m-Landsat Data 


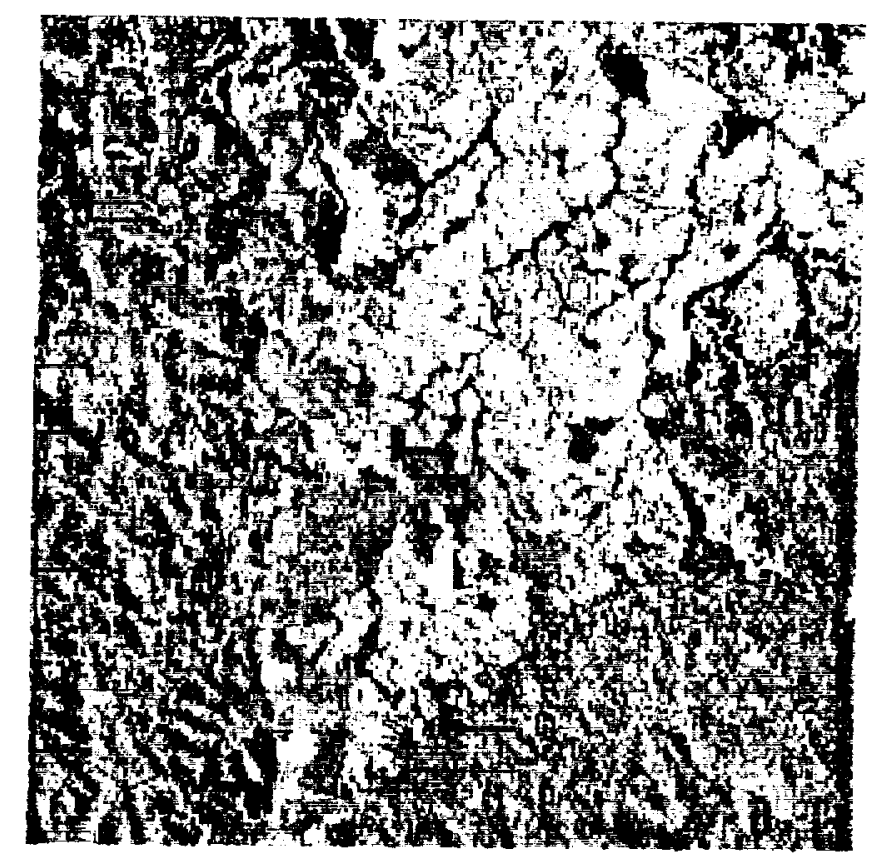

Figure 9

Resulting Fused Image from SAR and Landsat Data at a 6 Meters Resolution

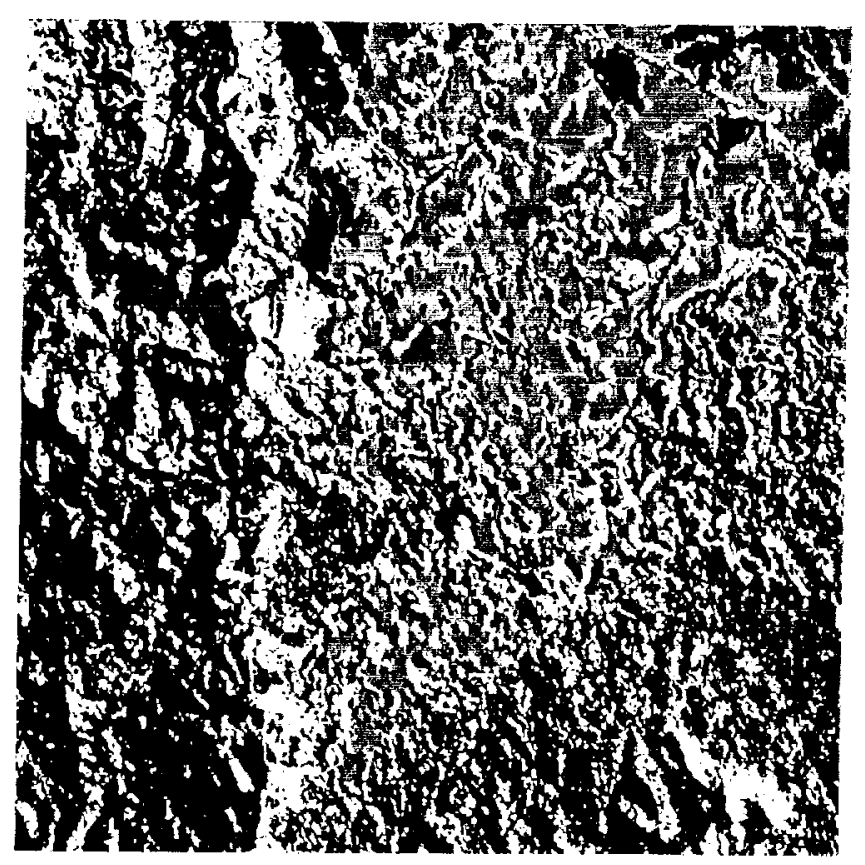

Figure 10

Clustering of Landsat Data

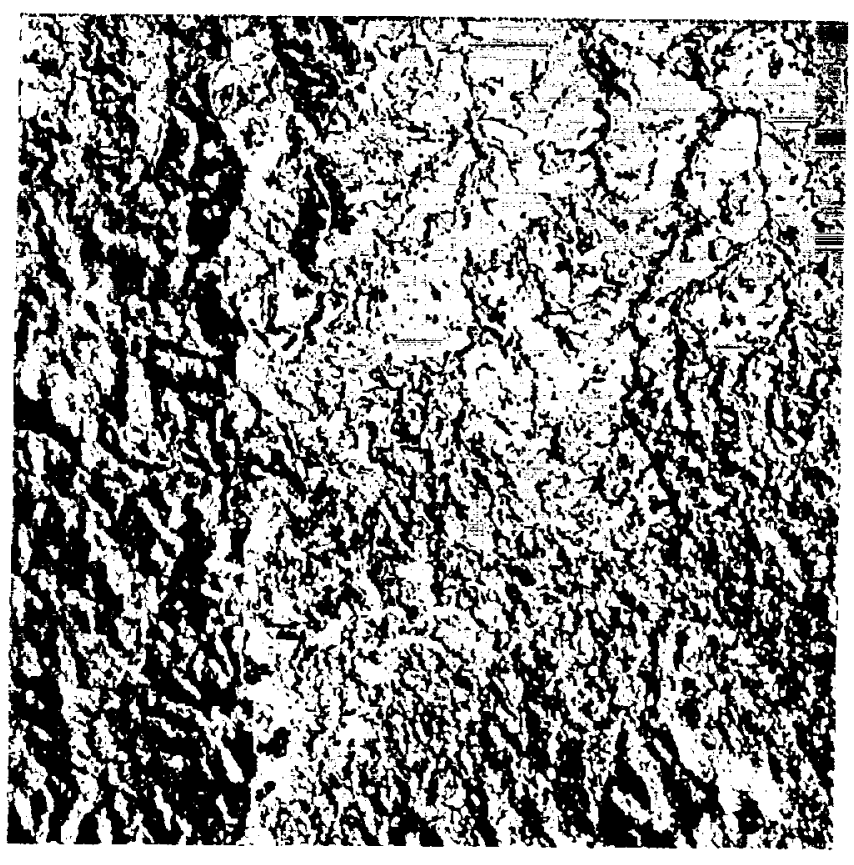

Figure 11

Clustering of Fused Data 